J. Gynäkol. Endokrinol. AT 2020 · 30:64-66 https://doi.org/10.1007/s41974-020-00141-z Online publiziert: 2. Juni 2020

(c) Der/die Autor(en) 2020, korrigierte

Publikation 2021

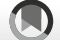

check tor
updates

\section{Marcus Schmidt}

Abteilung für Molekulare Onkologie, Klinik und Poliklinik für Geburtshilfe und Frauengesundheit,

Universitätsmedizin der Johannes Gutenberg-Universität Mainz, Mainz, Deutschland
Zielgerichtete Therapien werden beim Mammakarzinom seit vielen Jahren verwendet. Neben dem Nachweis der Hormonrezeptoren spielt der humane epidermale Wachstumsfaktor 2 (HER2) bei der Therapie des frühen wie auch des fortgeschrittenen Mammakarzinoms eine entscheidende Rolle [1,2]. Darüber hinaus hat eine verbesserte molekularbiologische Charakterisierung des Mammakarzinoms in der letzten Dekade zu neuen Angriffspunkten für eine gezielte Therapie geführt [3]. Dies erweitert die Möglichkeiten der zielgerichteten Therapie beim Mammakarzinom.

Beim sog. triple-negativen Mammakarzinom (TNBC), das negativ für den Östrogenrezeptor (ER), den Progesteronrezeptor (PR) und HER2 ist, ist in den letzten Jahren die Bedeutung des Immunsystems immer mehr in den Fokus des Interesses gerückt. Beim TNBC werden deutlich mehr somatische $\mathrm{Mu}$ tationen und Neoantigene nachgewiesen als bei anderen molekularen Subtypen, was für eine erhöhte Immunogenität spricht [4]. Tatsächlich konnte gezeigt werden, dass sowohl Transkripte von Immunzellen als auch tumorinfiltrierende Lymphozyten (TIL) ihren stärksten prognostischen und prädiktiven Einfluss

Vortragsmanuskript anlässlich der XXIX. Wissenschaftlichen Tagung der Arbeitsgemeinschaft für Gynäkologische Onkologie (AGO) der Österreichischen Gesellschaft für Gynäkologie und Geburtshilfe (OEGGG) 2020, 18. Österreichischer Kongress "Krebs bei der Frau“, 22.-25. April 2020, Salzburg. Die Tagung wurde aufgrund der SARS-CoV-2-Pandemie abgesagt. bei TNBC haben [5-7]. Mit dem Immuncheckpointinhibitor (ICPi) Atezolizumab konnte ein verbessertes progressionsfreies Überleben (PFS; 7,5 vs. 5 Monate; Hazard Ratio [HR] 0,62) sowie ein verlängertes Gesamtüberleben (OS; 25 vs. 15,5 Monate; HR 0,629) bei fortgeschrittenen TNBC mit Positivität für den „programmed cell death ligand 1"(PD-L1) gezeigt werden [8].

Ein weiterer prädiktiver Marker beim fortgeschrittenen Mammakarzinom ist der Nachweis von Mutationen im Breastcancer-1(BRCA1)- oder BRCA2-Gen in der Keimbahn der Patientinnen. Bei Nachweis einer BRCA-Mutation können Inhibitoren der Poly(ADP-Ribose)Polymerase (PARP) eingesetzt werden. Bei BRCA-mutierten Tumoren, die einen Defekt in der homologen Rekombination, die zur fehlerfreien Reparatur von DNA-Doppelstrangbrüchen dient, aufweisen, führt dies zur sogenannten synthetischen Letalität [9]. Durch die Blockade dieser beiden Reparaturmechanismen können die Tumorzellen DNASchäden nicht mehr effizient reparieren. Die PARP-Inhibitoren Olaparib ( 8,0 vs. 4,2 Monate; HR 0,58) und Talazoparib (8,6 vs. 5,6 Monate; HR 0,54$)$ verlängern das PFS bei Patientinnen mit einer BRCA-Mutation [10, 11]. Für Olaparib ist zwischenzeitlich auch eine OS-Verlängerung bei Patientinnen, die in der ersten Therapielinie behandelt wurden, nachgewiesen [12].

Bei Hormonrezeptor-positiven fortgeschrittenen Mammakarzinomen spielt der Phosphatidylinositol-3-Kinase(PI3K)
Weg eine wesentliche Rolle. Patientinnen mit Mutationen im PIK3CA-Gen zeigen ein signifikant verlängertes $\mathrm{PFS}$ (11 vs. 5,7 Monate; HR 0,65), wenn sie zusätzlich zu Fulvestrant noch mit dem PI3K-Inhibitor Alpelisib behandelt werden [13]. „Downstream" von PI3K liegt die Kinase $A K T$. Das PFS (10,3 vs. 4,8 Monate; HR 0,58) konnte durch Hinzunahme des AKT-Inhibitors Capivasertib bei Hormonrezeptorpositiven Patientinnen in der FAKTION-Studie signifikant verlängert werden [14]. Auch bei fortgeschrittenem TNBC führen AKT-Inhibitoren in der PAKTStudie zu einem verbesserten PFS (5,9 vs. 4,2 Monate; HR 0,74) und zeigen sogar eine Verlängerung des OS (19,1 vs. 12,6 Monate; HR 0,61; [15]). Diese Veränderungen waren bei Tumoren mit Alterationen im PIK3CA/AKT1/PTENWeg am deutlichsten (PFS 9,3 vs. 3,7 Monate; HR 0,14), sodass eine genauere molekularbiologische Charakterisierung in Zukunft immer wichtiger wird.

Beim fortgeschrittenen Mammakarzinom können Mutationen im Östrogenrezeptor (ESR1) nachgewiesen werden, die unter einer Behandlung mit Aromatasehemmern selektiert werden. In der SOFEA-Studie wiesen $39,1 \%$ und in der PALOMA-3-Studie 25,3\% eine ESR1Mutation auf. Mammakarzinome mit einer solchen ESR1-Mutation sprechen in retrospektiven Analysen prospektiver Studien besser auf eine Therapie mit Fulvestrant als auf einen Aromatasehemmer an [16]. Die Hazard Ratio lag zwischen 0,43 (PALOMA-3) und 0,52 (SOFEA). 
HER2-Mutationen treten unabhängig von einer HER2-Überexpression oder -Amplifikation in 2,4\% auf, wobei die Rate bei lobulären $(7,2 \%)$ höher ist als bei duktalen (1,6\%) Mammakarzinomen [17]. In einer kleinen Phase-IIStudie hatten stark vorbehandelte $\mathrm{Pa}$ tientinnen mit einer HER2-Mutation ein progressionsfreies Überleben (PFS) von 16 Wochen unter dem Tyrosinkinaseinhibitor Neratinib. Ähnlich wie bei einer ESR1- oder einer PK3CAMutation kann auch eine HER2-Mutation aus zirkulierender Tumor-DNA (ctDNA) nachgewiesen werden, was die Praktikabilität verglichen mit der $\mathrm{Mu}$ tationsanalyse aus einer Biopsie einer Metastase verbessert.

Seit Langem besteht eine große Hoffnung in einer verbesserten molekulargenetischen Charakterisierung, die durch den Nachweis von therapierbaren („actionable") Mutationen zu einer Präzisionsmedizin („precision medicine“) mit verbesserter Prognose bei fortgeschrittenen Krebserkrankungen führen soll. Das Konzept der Präzisionsmedizin ist überzeugend, allerdings liegt bislang erst eine prospektive, randomisierte Studie in der Onkologie vor. In der SHIVA-Studie untersuchten Le Tourneau und Mitarbeiter 741 fortgeschrittene Malignome ( 20\% Mammakarzinome) und konnten bei $40 \%$ molekulare Alterationen finden [18]. Die Patienten wurden dann randomisiert zwischen einer Standardtherapie und einer gezielten Therapie der molekularen Alterationen. Das PFS konnte allerdings durch die gezielte mutationsspezifische Therapie nicht signifikant verbessert werden (PFS 2,3 vs. 2,0 Monate; HR 0,88; $P=0,41$ ), sodass die Autoren aus ihren Ergebnissen schlussfolgerten, dass ein Einsatz von gezielten Therapien außerhalb der Zulassung nicht unterstützt werden kann. Dessen ungeachtet sind weitere mögliche Angriffspunkte für gezielte Therapien beim fortgeschrittenen Mammakarzinom, die allerdings in weniger als $1 \%$ der Mammakarzinome zu finden sind, zum einen der Nachweis einer Mikrosatelliteninstabilität (MSI), die zu einer deutlich erhöhten Rate an Mutationen führt [19], und zum anderen eine Neurotrophe-Tyrosinrezeptorkinase(NTRK)-Genfusion, die

J. Gynäkol. Endokrinol. AT 2020 · 30:64-66 https://doi.org/10.1007/s41974-020-00141-z (c) Der/die Autor(en) 2020

\section{Schmidt}

\section{Zielgerichtete Therapie beim metastasierten Mammakarzinom - welche molekularen Tests sind notwendig?}

\section{Zusammenfassung}

In den letzten Jahren ist die zielgerichtete Therapie beim Mammakarzinom immer mehr in den Fokus gerückt. Neben den Hormonrezeptoren und dem humanen epidermalen Wachstumsfaktor 2 (HER2) sind derzeit für die Festlegung einer gezielten Therapie vor allem der immunhistochemische Nachweis des „programmed cell death ligand 1" (PD-L1) bei fortgeschrittenen triplenegativen Mammakarzinomen sowie der Nachweis von Mutationen im Breast-cancer-
1(BRCA1)- oder BRCA2-Gen in der Keimbahn der Patientinnen und von Mutationen im Phosphatidylinositol-3-Kinase(PI3K)-Weg relevant.

\section{Schlüsselwörter}

Mammakarzinom · Programmed cell death ligand 1 (PD-L1) - Breast cancer (BRCA) 1/2 Phosphatidylinositol-4,5-bisphosphate 3kinase catalytic subunit alpha (PIK3CA) . Präzisionsonkologie

\section{Targeted Therapy in Metastatic Breast Cancer-Which Molecular Tests Are Necessary?}

\section{Abstract}

In recent years, targeted therapy for breast cancer has received increasingly more attention. In addition to hormone receptors and human epidermal growth factor 2 (HER2), the immunohistochemical detection of programmed cell death ligand 1 (PD-L1) in advanced triple-negative breast cancer and the detection of mutations in the breast cancer 1 (BRCA1) or BRCA2 gene in the patient's germline and mutations in the phosphatidylinositol 3 kinase (PI3K) pathway are currently relevant when making decisions regarding targeted therapy.

\section{Keywords}

Breast neoplasms - Programmed cell death ligand 1 (PD-L1) · Breast cancer (BRCA)1/2 Phosphatidylinositol-4,5-bisphosphate 3kinase catalytic subunit alpha (PIK3CA) . Precision oncology zumeist bei sekretorischen Mammakarzinomen beobachtet wird [20]. Sowohl für Malignome mit NTRK-Genfusion als auch mit Mikrosatelliteninstabilität können Therapien tumoragnostisch mit Erfolg eingesetzt werden [21, 22], wobei allerdings auch hier bislang noch randomisierte Evidenz aussteht.

Insgesamt haben die verbesserte molekularbiologische Charakterisierung und die Entwicklung neuer molekularer Tests, von denen der Nachweis von $P D-L 1$ sowie Mutationsanalysen von $B R C A$ und PIK3CA bereits als Standard betrachtet werden können, die therapeutischen Optionen beim fortgeschrittenen Mammakarzinom deutlich erweitert.

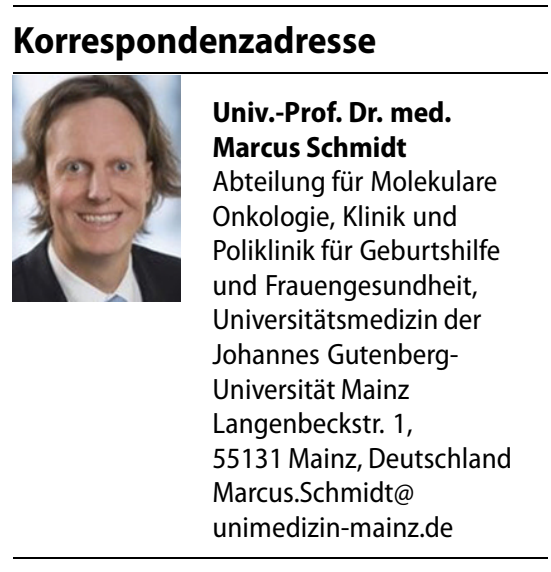

Funding. Open Access funding enabled and organized by Projekt DEAL

\section{Einhaltung ethischer Richtlinien}

Interessenkonflikt. M. Schmidt war als Referent für AstraZeneca, Celgene, Eisai, Novartis, Pfizer und Roche tätig und hat von diesen Firmen sowie von Lilly, Myelo 
Originalien

Therapeutics, Pantarhei Bioscience und Pierre Fabre auch Beraterhonorare erhalten. Forschungsunterstützung von AstraZeneca, BioNTech, Eisai, Genentech, Myelo Therapeutics, Novartis, Pantarhei Bioscience, Pfizer, Pierre Fabre und Roche.

Für diesen Beitrag wurden vom Autor keine Studien an Menschen oder Tieren durchgeführt. Für die aufgeführten Studien gelten die jeweils dort angegebenen ethischen Richtlinien.

Open Access Dieser Artikel wird unter der Creative Commons Namensnennung 4.0 International Lizenz veröffentlicht, welche die Nutzung, Vervielfältigung Bearbeitung, Verbreitung und Wiedergabe in jeglichem Medium und Format erlaubt, sofern Sie den/die ursprünglichen Autor(en) und die Quelle ordnungsgemäß nennen, einen Link zur Creative Commons Lizenz beifügen und angeben, ob Änderungen vorgenommen wurden.

Die in diesem Artikel enthaltenen Bilder und sonstiges Drittmaterial unterliegen ebenfalls der genannten Creative Commons Lizenz, sofern sich aus der Abbildungslegende nichts anderes ergibt. Sofern das betreffende Material nicht unter der genannten Creative Commons Lizenz steht und die betreffende Handlung nicht nach gesetzlichen Vorschriften erlaubt ist, ist für die oben aufgeführten Weiterverwendungen des Materials die Einwilligung des jeweiligen Rechteinhabers einzuholen.

Weitere Details zur Lizenz entnehmen Sie bitte de Lizenzinformation auf http://creativecommons.org/ licenses/by/4.0/deed.de.

\section{Literatur}

1. Burstein $H J$, Curigliano $G$, Loibl $S$, Dubsky $P$, Gnant M, Poortmans P, Colleoni M, Denkert C, Piccart-Gebhart M, Regan M, Senn $H$, Winer EP Thurlimann B (2019) Estimating the benefits of therapy for early-stage breast cancer: the St. Gallen International Consensus Guidelines for the primary therapy of early breast cancer 2019. Ann Oncol 30(10):1541-1557. https://doi.org/10. 1093/annonc/mdz235

2. Cardoso F, Senkus E, Costa A et al (2018) 4th ESO-ESMO International Consensus Guidelines for Advanced Breast Cancer (ABC 4). Ann Oncol 29(8):1634-1657. https://doi.org/10.1093/ annonc/mdy192

3. Hanahan D, Weinberg RA (2011) Hallmarks of cancer: the next generation. Cell 144(5):646-674. https://doi.org/10.1016/j.cell.2011.02.013

4. Narang $P$, Chen $M$, Sharma AA, Anderson KS, Wilson MA (2019) The neoepitope landscape of breast cancer: implications for immunotherapy. BMC Cancer 19(1):200. https://doi.org/10.1186/ s12885-019-5402-1

5. Adams S, Gray RJ, Demaria S, Goldstein L, Perez EA, Shulman LN, Martino $S$, Wang $M$, Jones VE, Saphner TJ, Wolff AC, Wood WC, Davidson NE, Sledge GW, Sparano JA, Badve SS (2014) Prognostic value of tumor-infiltrating lymphocytes in triple-negative breast cancers from two phase III randomized adjuvant breast cancer trials: ECOG 2197 and ECOG 1199. J Clin Oncol32(27):2959-2966.https://doi.org/10.1200/ JCO.2013.55.0491

6. Denkert C, von Minckwitz G, Brase JC, Sinn BV Gade S, Kronenwett R, Pfitzner BM, Salat C,
Loi S, Schmitt WD, Schem C, Fisch K, DarbEsfahani S, Mehta K, Sotiriou C, Wienert S, Klare P, André $\mathrm{F}$, Klauschen $\mathrm{F}$, Blohmer J, Krappmann $\mathrm{K}$, Schmidt M, Tesch H, Kümmel S, Sinn P, Jackisch C, Dietel M, Reimer T, Untch M, Loibl S (2015) Tumor-infiltrating lymphocytes and response to neoadjuvant chemotherapy with or without carboplatin in human epidermal growth factor receptor 2-positive and triple-negative primary breast cancers. J Clin Oncol 33(9):983-991. https:// doi.org/10.1200/JCO.2014.58.1967

7. Schmidt $M$, Weyer-Elberich $V$, Hengstler JG, Heimes A, Almstedt K, Gerhold-Ay A, Lebrecht A, Battista MJ, Hasenburg A, Sahin U, Kalogeras KT, Kellokumpu-Lehtinen P, Fountzilas G, Wirtz RM Joensuu H (2018) Prognostic impact of CD4positive Tcell subsets in early breast cancer: a study based on the FinHer trial patient population. Breast Cancer Res 20(1):15. https://doi.org/10. 1186/s13058-018-0942-x

8. Schmid P, Adams $S$, Rugo HS, Schneeweiss $A$, Barrios $\mathrm{CH}$, Iwata $\mathrm{H}$, Dieras $\mathrm{V}$, Hegg $\mathrm{R}$, Im $\mathrm{S}$, Shaw Wright G, Henschel V, Molinero L, Chui SY, Funke R, Husain A, Winer EP, Loi S, Emens LA (2018) Atezolizumab and nab-paclitaxel in advanced triple-negative breast cancer. $\mathrm{N}$ Engl J Med 379(22):2108-2121. https://doi.org/10.1056/ NEJMoa1809615

9. Ashworth A (2008) A synthetic lethal therapeutic approach: poly(ADP) ribose polymerase inhibitors for the treatment of cancers deficient in DNA double-strand break repair. J Clin Oncol 26(22):3785-3790. https://doi.org/10.1200/JCO. 2008.16.0812

10. Robson M, Im S, Senkus E, Xu B, Domchek SM, Masuda N, Delaloge S, Li W, Tung N, Armstrong A, Wu W, Goessl C, Runswick S, Conte P (2017) Olaparib for metastatic breast cancer in patients with a germline BRCA mutation. $\mathrm{N}$ Engl $J$ Med 377(6):523-533. https://doi.org/10.1056/ NEJMoa 1706450

11. Litton JK, Rugo HS, Ettl J, Hurvitz SA, Gonçalves A Lee $K$, Fehrenbacher $L$, Yerushalmi R, Mina LA Martin M, Roché $H$, Im Y, Quek RGW, Markova D, Tudor IC, Hannah AL, Eiermann W, Blum JL (2018) Talazoparib in patients with advanced breast cancer and a germline BRCA mutation. $\mathrm{N}$ Engl J Med 379(8):753-763. https://doi.org/10.1056/ NEJMoa1802905

12. Robson ME, Tung $N$, Conte $P$, Im $S$, Senkus $E$ Xu B, Masuda N, Delaloge S, Li W, Armstrong A, Wu W, GoessI C, Runswick S, Domchek SM (2019) OlympiAD final overall survival and tolerability results: olaparib versus chemotherapy treatment of physician's choice in patients with a germline BRCA mutation and HER2-negative metastatic breast cancer. Ann Oncol 30(4):558-566. https:// doi.org/10.1093/annonc/mdz012

13. André $F$, Ciruelos $E$, Rubovszky $G$, Campone $M$, Loibl S, Rugo HS, Iwata H, Conte P, Mayer IA, Kaufman B, Yamashita T, Lu Y, Inoue K, Takahashi M, Pápai Z, Longin A, Mills D, Wilke C, Hirawat S, Juric D (2019) Alpelisib for PIK3CA-mutated, hormone receptor-positive advanced breast cancer. $\mathrm{N}$ Engl $J$ Med 380(20):1929-1940. https://doi.org/10.1056/ NEJMoa1813904

14. Jones RH, Casbard A, Carucci M, Cox C, Butler R, Alchami F, Madden T, Bale C, Bezecny P, Joffe J, Moon S, Twelves C, Venkitaraman R, Waters $S$, Foxley A, Howell SJ (2020) Fulvestrant plus capivasertib versus placebo after relapse or progression on an aromatase inhibitor in metastatic, oestrogen receptor-positive breast cancer (FAKTION): a multicentre, randomised, controlled, phase 2 trial.
Lancet Oncol 21(3):345-357. https://doi.org/10. 1016/S1470-2045(19)30817-4

15. SchmidP, Abraham J, ChanS, WheatleyD, Brunt AM, Nemsadze G, Baird RD, Park YH, Hall PS, Perren T, Stein RC, Mangel L, Ferrero J, Phillips M, Conibear J, Cortes J, Foxley A, de Bruin EC, McEwen R, Stetson D, Dougherty B, Sarker S, Prendergast A, McLaughlinCallan M, Burgess M, Lawrence C, Cartwright H, Mousa K, Turner NC (2020) Capivasertib plus paclitaxel versus placebo plus paclitaxel as firstline therapy for metastatic triple-negative breast cancer: the PAKT trial. J Clin Oncol 38(5):423-433. https://doi.org/10.1200/JCO.19.00368

16. Fribbens C, O'Leary B, Kilburn L, Hrebien S, GarciaMurillas I, Beaney M, Cristofanilli M, Andre F, Loi S, Loibl S, Jiang J, Bartlett CH, Koehler M, Dowsett M, Bliss JM, Johnston SRD, Turner NC (2016) Plasma ESR1 mutations and the treatment of estrogen receptor-positive advanced breast cancer. J Clin Oncol34(25):2961-2968.https://doi.org/10.1200/ JCO.2016.67.3061

17. Ma CX, Bose $R$, Gao F, Freedman RA, Telli ML, Kimmick G, Winer $E$, Naughton M, Goetz MP, Russell C, Tripathy D, Cobleigh M, Forero A, Pluard TJ, Anders C, Niravath PA, Thomas $S$, Anderson J, Bumb C, Banks KC, Lanman RB, Bryce R, Lalani AS, Pfeifer J, Hayes DF, Pegram M, BlackwellK, Bedard PL, Al-Kateb H, Ellis MJC (2017) Neratinib efficacy and circulating tumor DNA detection of HER2 mutations in HER2 nonamplified metastatic breast cancer. Clin Cancer Res 23(19):5687-5695. https://doi.org/10.1158/1078-0432.CCR-17-0900

18. Le Tourneau C, Delord J, Gonçalves A, Gavoille C, Dubot $C$, Isambert N, Campone M, Trédan 0 , Massiani M, Mauborgne C, Armanet S, Servant N, Bièche $I$, Bernard $V$, Gentien $D$, Jezequel $P$ Attignon $V$, Boyault $S$, Vincent-Salomon $A$ Servois V, Sablin M, Kamal M, Paoletti X (2015) Molecularly targeted therapy based on tumour molecular profiling versus conventional therapy for advanced cancer (SHIVA): a multicentre, openlabel, proof-of-concept, randomised, controlled phase 2 trial. Lancet Oncol 16(13):1324-1334. https://doi.org/10.1016/S1470-2045(15)00188-6

19. Dudley JC, Lin M, Le DT, Eshleman JR (2016) Microsatellite instability as a biomarker for PD-1 blockade. Clin Cancer Res 22(4):813-820. https:// doi.org/10.1158/1078-0432.CCR-15-1678

20. Remoué A, Conan-Charlet V, Bourhis $A$, Le Flahec G, Lambros L, Marcorelles P, Uguen A (2019) Non-secretory breast carcinomas lack NTRK rearrangements and TRK protein expression. Pathol Int 69(2):94-96. https://doi.org/10.1111/ pin. 12766

21. Drilon A, Laetsch TW, Kummar Setal (2018) Efficacy of larotrectinib in TRK fusion-positive cancers in adults and children. N Engl J Med 378(8):731-739. https://doi.org/10.1056/NEJMoa1714448

22. Lemery S, Keegan P, Pazdur R (2017) First FDA approval agnostic of cancer site-when a biomarker defines the indication. $\mathrm{N}$ Engl J Med377(15):1409-1412.https://doi.org/10.1056/ NEJMp1709968

Hinweis des Verlags. Der Verlag bleibt in Hinblick auf geografische Zuordnungen und Gebietsbezeichnungen in veröffentlichten Karten und Institutsadressen neutral. 